\title{
Cell detachment rapidly induces changes in noncoding RNA expression in human mesenchymal stromal cells
}

$\overline{\text { Elena Della Bella' \& Martin J Stoddart*,1 }}$

\section{ABSTRACT}

Aims: To identify differential expression of noncoding RNAs after trypsinization in human mesenchymal stromal cells (hMSCs), focusing on miRNAs, piRNAs and circRNAs. Methods: hMSCs from the bone marrow of three donors were collected for RNA extraction, either lysed directly in monolayer or trypsinized and lysed within $30 \mathrm{~min}$. Total RNA was isolated and sequenced for the evaluation of miRNA and piRNA expression or RNaseR treated and labeled for circRNA array hybridization. RT-qPCR was performed to evaluate the stability of candidate reference genes. Results \& conclusions: Alterations in levels of several noncoding RNAs are rapidly induced after trypsinization of hMSCs, affecting critical pathways. This should be carefully considered for a proper experimental design.

\section{METHOD SUMMARY}

Human mesenchymal stromal cells were expanded, and RNA collected from either trypsinized or monolayer cells for RNA sequencing or RNA microarray analysis. Differentially expressed miRNA, piRNA and circRNA were identified. Real-time PCR was used to validate miRNA and circRNA expression. Primers specific for circular RNA were designed with a divergent primer strategy or with one of the primers being specific for the backspliced junction. Finally, a stability analysis with algorithms as geNorm, NormFinder and the comparative $\Delta \mathrm{Ct}$ method allowed the identification of the most suitable reference genes for a proper gene expression analysis.

\section{KEYWORDS}

cell detachment $\cdot$ circular RNA $\cdot$ human mesenchymal stem cells $\cdot$ loss of anchorage - microRNA - noncoding RNA - piwi-RNA

- reference gene stability $\cdot$ RNA sequencing - trypsinization

'AO Research Institute, AO Foundation, Davos, Switzerland; *Author for correspondence: martin. stoddart@aofoundation.org

BioTechniques 67: 286-293 (December 2019) 10.2144/ btn-2019-0038
Detachment of anchorage-dependent cells from culture substrates may lead to profound changes in cell biology including, but not limited to, alteration of signaling pathways [1], oxygen consumption rates [2], and cytoskeleton reorganization [3]. Cytoskeletal tension influences nuclear shape and events [4,5]. Different methods of detachment also induce different effects on the cells, as in the expression of surface antigen [6] or in cell phonotype and function [7]. Altogether, it is evident that cell detachment from its substrate has deep consequences on the mechanobiology of cells that, in the end, may result in alterations of gene expression. This is particularly important to keep in mind for the proper design of experiments aimed at the evaluation of the expression of single genes or in transcriptomic analyses.

Transcriptomics has benefited from the rise of next-generation sequencing applications, including RNA sequencing. Indeed, compared with other techniques, no prior knowledge of sequences is needed, and this can also increase the body of knowledge by the prediction and identification of novel RNA species that were previously unrecognized.

The aim of this study was to identify noncoding RNAs that were rapidly differentially expressed after loss of anchorage in human bone marrow-mesenchymal stromal cells. In this study we focused on microRNA (miRNA), piwi-interacting RNA (piRNA) and circular RNA (circRNA).

miRNA represents the most known class of small noncoding RNAs and is well characterized in its role for the regulation of gene expression, and its relevance in several physiologic processes and diseases has been unraveled [8-12]. Other noncoding RNAs are less well known and characterized, but there is a growing interest in their identification and characterization as they have crucial roles in homeostasis and disease.

piRNAs [13] are the largest class of small noncoding RNAs, with their main function of repressing the expression of transposable elements in the germline by epigenetic silencing. piRNAs are also thought to regulate protein-coding gene expression in somatic cells, as the $3^{\prime}$ UTR of genes can be a source of piRNA and an important role in mouse spermatogenesis has been described [13-16]. As the name suggests, they interact with the PIWI subfamily of Argonaute proteins, leading to the formation of RNA-induced silencing complexes (RISCs) known as piRISCs. piRNAs are longer in length than miRNAs (26-31 nucleotides) and with a different biogenesis, which is independent of Dicer. A growing number of works report an important role for piRNA in cancer development [17] and in stem cell biology [18,19].

Previously thought to be artefacts or resulting from errors in RNA splicing, circRNA are highly stable, single-stranded RNAs that were recently recognized with regulatory functions $[20,21]$ and potentially translated into proteins [22]. They are generated from precursor mRNAs which, in a process known as backsplicing, are covalently closed at their ends producing the typical circular structure. Compared with miRNAs and piRNAs, circRNAs are longer and more complex in structure, and may contain one or more exons or even introns. As with the other noncoding RNAs, circRNAs regulate expression of genes through a number of mechanisms. So far, the following have been suggested: miRNA sequestration (the so-called 'miRNA sponges') acting as decoys in place of target mRNA; competition with the linear counterpart for RNA-binding 
- proteins; and impact on linear mRNA biogenesis (so altering the abundance of specific linear protein-coding RNAs).

Finally, a proper analysis of relative gene expression results by real-time PCR relies on the identification of stable reference genes to be used as internal controls; this allows the normalization of the gene or genes of interest on a set of endogenous markers that do not change expression among the experimental groups under study [23]. Commonly used reference genes, such as GAPDH or ACTB, are still thoroughly used for normalization of gene expression without being properly tested. However, it is recognized that those do not represent good 'housekeeping' genes in many cases because they may be regulated in the conditions under study, and no universal reference genes exist. The most appropriate set of reference genes should be tested and used for each application. Indeed, a growing number of papers focuses on this fundamental, but often overlooked, aspect of the analysis. For this reason, a panel of ten candidate reference genes was screened for stability using three commonly employed algorithms, revealing the most stable single reference genes and their possible combination to be used in the study of cell adhesion and loss of anchorage.

\section{MATERIALS \& METHODS}

Cell isolation \& culture

Human mesenchymal stromal cells (hMSCs) were isolated from bone marrow as previously described [24]. Bone marrow aspirates were harvested during routine procedures from the vertebral body of three patients (\#1: male 59 years; \#2: male 80 years; \#3: female 55 years), obtained with full ethical approval (Bern Req-2016-00141). After isolation, hMSCs were subcultured maintaining an initial cell density of $3 \times 10^{3}$ cells $/ \mathrm{cm}^{2}$ and grown until passage 2 in $\alpha$-MEM (Gibco, Thermo Fisher, Zürich, Switzerland) with the addition of $10 \%$ MSC-qualified FBS (Pan-Biotech, Aidenbach, Germany), $100 \mathrm{U} / \mathrm{ml}$ penicillin, $100 \mu \mathrm{g} / \mathrm{ml}$ streptomycin (Gibco) and $5 \mathrm{ng} / \mathrm{ml}$ bFGF (Peprotech, NJ, USA). Cultures were maintained at $37^{\circ} \mathrm{C} / 5 \% \mathrm{CO}_{2}$, and the medium was refreshed every second day.

At p.2, cells were further expanded in the same expansion medium. When the cells were $70 \%$ confluent, cells were collected for RNA extraction. Monolayer of cells in triplicate, grown in $25 \mathrm{~cm}^{2}$ flasks (day 0 2D), were lysed directly with $1 \mathrm{ml}$ of TRIreagent (Molecular Research Center Inc., $\mathrm{OH}$, USA). The other cells were washed with phosphate-buffered saline and detached from substrate with an incubation with $0.05 \%$ Trypsin-EDTA (Gibco, Thermo Fisher) for $3 \mathrm{~min}$ at $37^{\circ} \mathrm{C}$. The action of the enzyme was inhibited by the addition of 2 volumes of $10 \%$ FBS-containing medium. After centrifugation at $300 \times \mathrm{g}$ for $5 \mathrm{~min}$ at room temperature, the supernatant was removed and the cell pellet resuspended in growth medium. The cells were then counted with a handheld cell counter (Scepter 2.0, Merck Millipore, Darmstadt, Germany). For each donor, three different aliquots of $1 \times 10^{6}$ cells each (day 0 3D) were lysed in $1 \mathrm{ml}$ of TRIreagent within 30 min of trypsinization. Total number of samples: 18 (three donors, two conditions in triplicate).

\section{RNA isolation}

Total RNA was isolated from day $02 \mathrm{D}$ and 3D samples collected in TRIreagent following manufacturer's instructions. Briefly, $100 \mu \mathrm{l}$ of 1-Bromo-3-chloropropane (Sigma-Aldrich, MO, USA) was added to TRIreagent, mixed thoroughly and the samples were centrifuged at $12,000 \times g$ for $15 \mathrm{~min}$ at $4^{\circ} \mathrm{C}$. The recovered aqueous phase was mixed with $500 \mu \mathrm{l}$ of 2-propanol (Sigma-Aldrich) and precipitation of RNA was carried out overnight at $-20^{\circ} \mathrm{C}$ for enhanced recovery of small RNA species. The RNA pellet was then washed with $75 \% \mathrm{EtOH}$ (Sigma-Aldrich) and resuspended in $20 \mu$ of DEPC-treated $\mathrm{H}_{2} \mathrm{O}$. Total RNA concentration was measured with NanoDrop 1000 (Thermo Fisher) and purity was assessed by evaluation of the A260/280 and $\mathrm{A} 260 / 230$ ratios.

\section{RNAseq \& hybridization}

Two out of three replicates for each donor and sample group were selected for sequencing ( $n=12$ samples in total). A total of $2-\mu \mathrm{g}$ of total RNA for each sample was collected and dried in RNAstable ${ }^{m}$ tubes (Biomatrica, CA, USA) following the manufacturer's instructions. Samples were sent to Arraystar (MD, USA) for library generation and sequencing. The libraries were denatured as ssDNA molecules, captured on Illumina flow cells, amplified in situ as sequencing clusters and sequenced for 51 cycles on Illumina NextSeq500 system per the manufacturer's instructions.

For miRNA, trimmed reads were aligned to the reference genome and differentially expressed miRNA calculated. The novel miRNAs were also predicted. Hierarchical clustering, miRNA target analysis, target gene ontology, target pathway analysis, scatter plots and volcano plots were performed for the differentially expressed miRNA in R or Python environments for statistical computing and graphics.

For piRNA, trimmed reads were aligned to the piRBase [25]. The maximum mismatch $\leq 2$ reads were kept. The alignment statistical analysis was applied to retain the valid sequences for piRNA expression profiling and differential expression analysis. Hierarchical clustering, scatter plots and classification analysis were performed for the differentially expressed piRNA in R or Perl environments for statistical computing and graphics.

For circRNA, total RNAs were digested with RNase R (Epicentre Inc., WI, USA) to remove linear RNAs and enrich circRNAs, which were amplified and transcribed into fluorescent cRNA utilizing a random priming method (Arraystar Super RNA Labeling Kit; Arraystar). The labeled cRNAs were hybridized onto the Arraystar Human circRNA Array V2 (8x15K, Arraystar). After having washed the slides, the arrays were scanned by the Agilent Scanner G2505C. Agilent Feature Extraction software (version 11.0.1.1) was used to analyze acquired array images. Quantile normalization and subsequent data processing were performed using the $\mathrm{R}$ software limma package. Differentially expressed circRNAs with statistical significance between two groups were identified through volcano plot filtering. Differentially expressed circRNAs between two samples were identified through fold change filtering. Hierarchical clustering was performed to show the distinguishable circRNA expression pattern among samples.

\section{cDNA synthesis \& qPCR for} analysis of reference gene stability For the analysis of reference gene stability, reverse transcription and qPCR were performed using our standard protocol for 
the amplification of total RNA-derived cDNA. cDNA was synthesized using TaqMan reverse transcription reagents (Applied Biosystems, CA, USA), using 500 ng of starting RNA and following the manufacturer's instruction. cDNA was diluted 1:10 with TE buffer prior to downstream analyses.

The expression of 10 candidate reference genes was assessed by real-time PCR in a 384-well plate. A total of $5 \mathrm{ng}$ of cDNA in $2 \mu \mathrm{l}$ were used in each reaction. No template controls and no RT controls were included in the plate. Amplification of target genes was achieved using a TaqMan Gene Expression Master Mix (Applied Biosystems) in a QuantStudio 6 Flex Real-Time PCR System (Applied Biosystems) with the following protocol: $2 \mathrm{~min}$ at $50^{\circ} \mathrm{C} ; 10 \mathrm{~min}$ at $95^{\circ} \mathrm{C} ; 40$ cycles of $15 \mathrm{~s}$ at $95^{\circ} \mathrm{C}, 1 \mathrm{~min}$ at $60^{\circ} \mathrm{C}$.

The calculated $\mathrm{Ct}$ values were used for analysis of housekeeping stability in order to select the most suitable reference gene(s) for normalization of coding gene expression.

The list of reference genes together with assay information can be found in Supplementary Table 1. The genes were tested using TaqMan gene expression assay (Thermo Fisher), except for RPLPO, whose sequences were synthesized by Microsynth AG (Balgach, Switzerland). The primers used for gene expression analysis of RPLPO, which encodes for ribosomal protein lateral stalk subunit $P 0$, were as follows: forward primer: 5'-TGGGCAAGAACACCATGATG-3'; reverse primer: 5'-CGGATATGAGGCAGCAGTTTC-3'; probe sequence: 5'-AGGGCACCTGGAAAACAACCCAGC-3'; probe modification: 5'-FAM; 3'-TAMRA.

\section{Analysis of candidate reference genes}

The stability of candidate reference genes for coding gene expression analysis (Supplementary Table 1) was evaluated as previously described [26]. Three different algorithms have been applied, namely geNorm ([23], using v.3 Excel based macro), NormFinder ([27], using v.0.953 Excel add-in) and the comparative $\Delta \mathrm{Ct}$ method [28]. For data input in geNorm and NormFinder, the $Q$ value for each gene was calculated as $\mathrm{Q}=2^{-\Delta \mathrm{Ct}}$, where $\Delta \mathrm{Ct}$ is the difference between the $\mathrm{Ct}$ calculated from each sample and the one with the lowest value.

The same approach, with geNorm and NormFinder analysis, was also applied for the stability analysis of miRNA used as an endogenous control (hsa-miR-361-5p, hsa-miR-186-5p and their geometric mean, as described in the next section).

\section{Target validation}

For circRNA analysis, all the RNA samples $(n=18)$ were first digested with RNase $R$ ( 10 min at $37^{\circ} \mathrm{C}, 1 \mathrm{U}$ enzyme/ $1 \mu \mathrm{g}$ RNA) and immediately reverse transcribed into cDNA using the SuperScript VILO cDNA Synthesis Kit (Thermo Fisher) according to the manufacturer's protocol. Primers for circRNA detection were designed using NCBI's PrimerBLAST [29], with particular care taken to avoid the detection of unwanted targets (both the parental linear transcript or other sequences). The strategy for the design of the primers was that of divergent primers or with one primer specific for the backspliced junction, as suggested in the paper by Huang et al. [21]. Primer sequences and other details are reported in Supplementary Table 2. qPCR for circRNA detection in SYBR green was performed using Power SYBR Green PCR Master Mix (Thermo Fisher) in a QuantStudio 6 Flex Real-Time PCR System (Applied Biosystems) with the following protocol: initial denaturation for $10 \mathrm{~min}$ at $95^{\circ} \mathrm{C} ; 40$ cycles of $15 \mathrm{~s}$ at $95^{\circ} \mathrm{C}$ and $1 \mathrm{~min}$ at $60^{\circ} \mathrm{C}$. The protocol was concluded with a melt curve analysis to check for amplicon specificity. Results were calculated as $2^{-\Delta C t}$, using RPLPO as a reference gene (forward and reverse primer as described above).

For miRNA analysis, $10 \mathrm{ng}$ of total RNA was used for miRNA-specific reverse transcription using the TaqMan Advanced miRNA cDNA Synthesis Kit (Thermo Fisher), which uses a universal RT chemistry to reverse transcribe and preamplify all mature miRNAs by prior 3' poly-A tailing and 5'-adaptor ligation. Then, after a 1:10 dilution of the cDNA template, qPCR was performed using TaqMan Fast Advanced Master Mix (Thermo Fisher) for the detection of the following miRNAs, which were identified as differentially regulated from RNAseq analysis: hsa-miR-205-5p, hsa-miR-429, hsa-miR-1246, hsa-miR-197-3p, hsa-miR1298-5p, hsa-miR-200b-3p, hsa-miR-200c-3p, hsa-miR-494-5p, hsa-miR-9-3p and hsa-miR16-1-3p. hsa-miR-361-5p and hsa-miR-186-5p were used as endogenous controls with preliminary stability analysis. Assay details are included in Supplementary Table 3. The results were calculated as $2^{-\Delta c t}$, with normal- ization on the Ct geometric mean between hsa-miR-361-5p and hsa-miR-186-5p.

\section{Statistical analysis}

GraphPad Prism v.8 (GraphPad Software, CA, USA) was used for statistical analysis. A two-tailed unpaired Student's t-test was used to compare gene expression levels between day $02 \mathrm{D}$ and day 0 3D samples. Welch's correction was applied to the unpaired t-test for the comparison of hsa-miR-1246 between groups, since variances were significantly different $(p=0.0012)$.

\section{RESULTS \& DISCUSSION}

Differential expression of miRNA

From RNAseq analysis, differentially expressed RNAs were identified with a p-value cutoff of 0.05 (Supplementary Table 4). A total of 36 miRNAs were upregulated, while seven were downregulated in suspension cells (day 0_3D) compared with those in adhesion (day 0_2D). The false discovery rate (FDR) calculated with the Benjamini-Hochberg method is also reported. Highlighted in bold are the miRNAs that showed also a low FDR. Figure 1 shows the pathway enrichment analysis results of both up- and downregulated miRNAs.

miRNA raw data from sequencing analysis are available in the Gene Expression Omnibus (GEO) database (accession number: GSE129345) [30].

\section{Differential expression of} piwi-interacting RNAs

RNAseq identified several differentially regulated piRNAs in $3 D$ versus $2 D$ cells. More specifically, 48 of them were upregulated, while 14 were downregulated as identified by $p$-values. A few piRNAs (five within the upregulated and two within the downregulated ones) also showed a very low FDR and are highlighted in bold in Supplementary Table 5. The table also indicates the references where the piRNAs were previously identified [31-35].

piRNA raw data from sequencing analysis are available in the GEO database (accession number: GSE129350) [36].

\section{Differential expression of circRNA}

Ten different circRNAs have been identified as differentially upregulated in $3 D$ vs $2 D$ day 0 cells $(p<0.05)$, as indicated in 


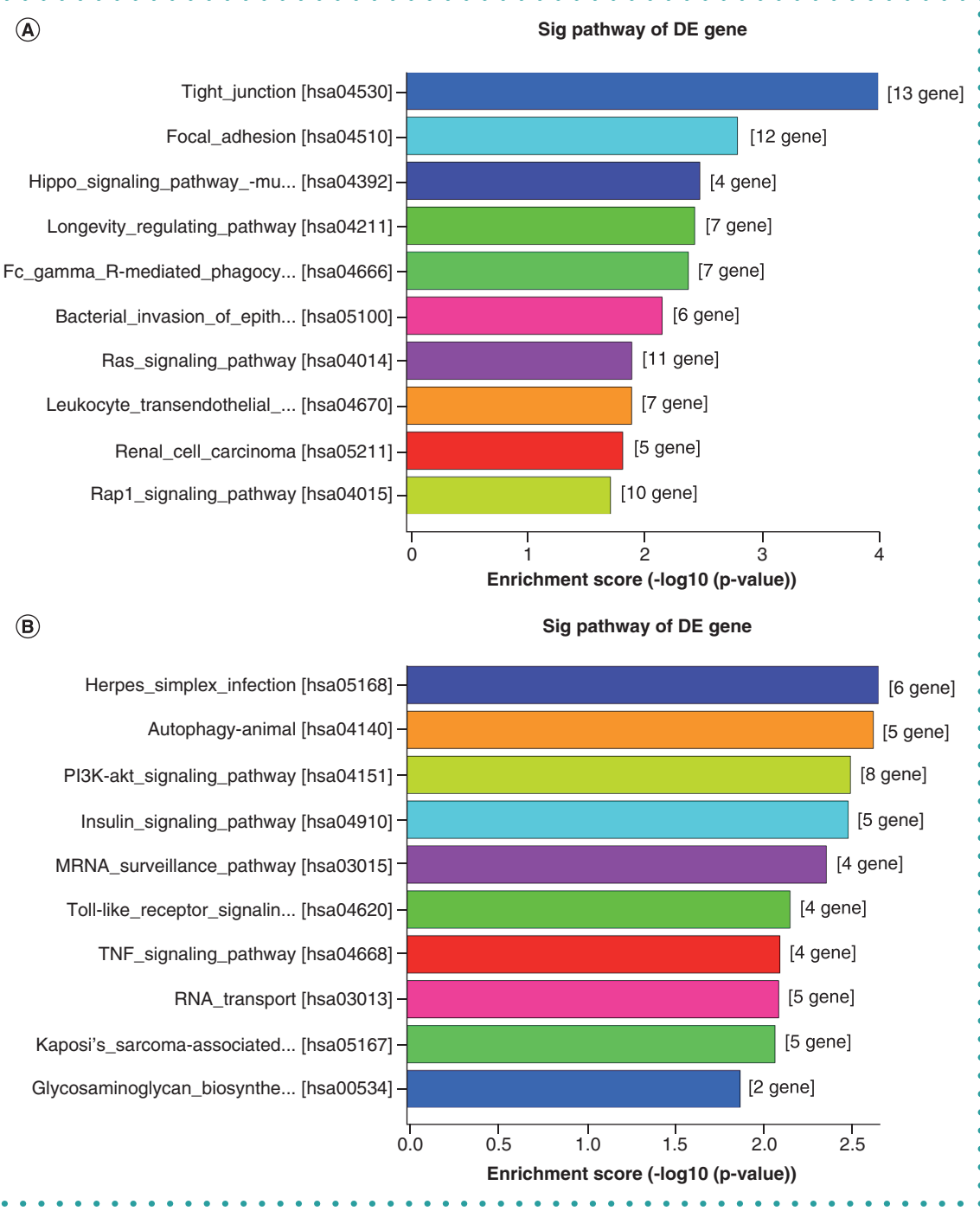

Figure 1. Pathway Enrichment Score analysis of differential miRNA expression in trypsinized versus adherent human mesenchymal stromal cells. (A) Pathway Enrichment Score analysis for upregulated miRNAs. (B) Pathway Enrichment Score analysis for downregulated miRNAs.

> Supplementary Table 6. However, the FDR for all of them was $>0.99$, thus the probability that some of them are false positives is very high. No circRNA appears to be downregulated within $30 \mathrm{~min}$ from detachment. Supplementary Table 7 reports the differentially expressed miRNAs that are targets of upregulated circRNAs in our samples.

circRNA raw data from microarray analysis are available in the GEO database (accession number: GSE129357) [37].

\section{Candidate reference gene stability}

Overall, the reference genes tested for coding gene-expression analysis showed a high stability across conditions. The most stable reference genes are HPRT1, PGK1 and
$T B P$, as identified by the three algorithms (Supplementary Table 8). All the genes are, however, stable, since there is no massive variation and the stability values as defined by the three methods are very low (the lower the value is, the higher the stability). The best combination of reference genes identified by geNorm was HPRT1-TBP, while by NormFinder it was HPRT1-PGK1 (this is the only algorithm used that considers intergroup variation). Figure 2A depicts the range of variability of $\mathrm{Ct}$ values for the ten candidate reference genes, while Figure $2 \mathrm{~B}$ represents the average stability values of reference genes as calculated by geNorm.

For normalization of miRNA expression, both hsa-miR-361-5p and hsa-miR-186-5p showed a high stability between the two groups, as calculated by geNorm and NormFinder (Supplementary Table 9). However, their geometric average proved to be even more stable, thus this was used for normalization of target miRNA expression.

\section{Target validation}

Figure 3 summarizes the results of validation experiments on a selection of miRNAs, while the results on circRNA validation are reported in Figure 4 . The analysis confirmed the downregulation of hsa-miR-1246: from qPCR analysis it was 31.25 times less expressed in trypsinized versus adherent cells (day $03 \mathrm{D}$ vs day $02 \mathrm{D}$ fold change: 0.032 ), with $p<0.05$. The upregulation of hsa-miR-16-1-3p was also confirmed, with a fold change of 2.41 between $3 D$ versus $2 D$ samples $(p<0.05)$. The other miRNA tested were unchanged between the two groups or barely detectable, as in the case of hsa-miR1298-5p and hsa-miR-429 (data not shown).

For circRNA, we tested the expression of hsa_circRNA_104574 (derived from the TRIM35 gene), hsa_circRNA_100395 (KLHL20), hsa_circRNA_101015 (LRP6), hsa_circRNA_405396 (SCAPER) and hsa_ circRNA_023461 (ARAP1). Even though changes were seen in some donors in circRNA expression, the differences observed between the two groups did not reach significance, as depicted in Figure 4. While it is possible that their expression does indeed change, it might not be consistent between primary human donors.

While most protocols suggest direct cell lysis for RNA isolation of adherent cells, detachment by trypsinization or scraping off the monolayer is still used prior to the addition of cell lysis buffers. However, if gene expression of anchorage-dependent cells is to be analyzed, this may potentially lead to alterations in the cell transcriptome that are not correlated to the real research question under investigation. Here we show that loss of adherence induced by trypsinization of hMSCs indeed rapidly induces (within $30 \mathrm{~min}$ from detachment) a differential regulation of noncoding RNAs, such as miRNA, piRNA and circRNA.

Unsurprisingly, pathways identified from RNAseq for differentially regulated miRNAs are enriched in tight junctions and 


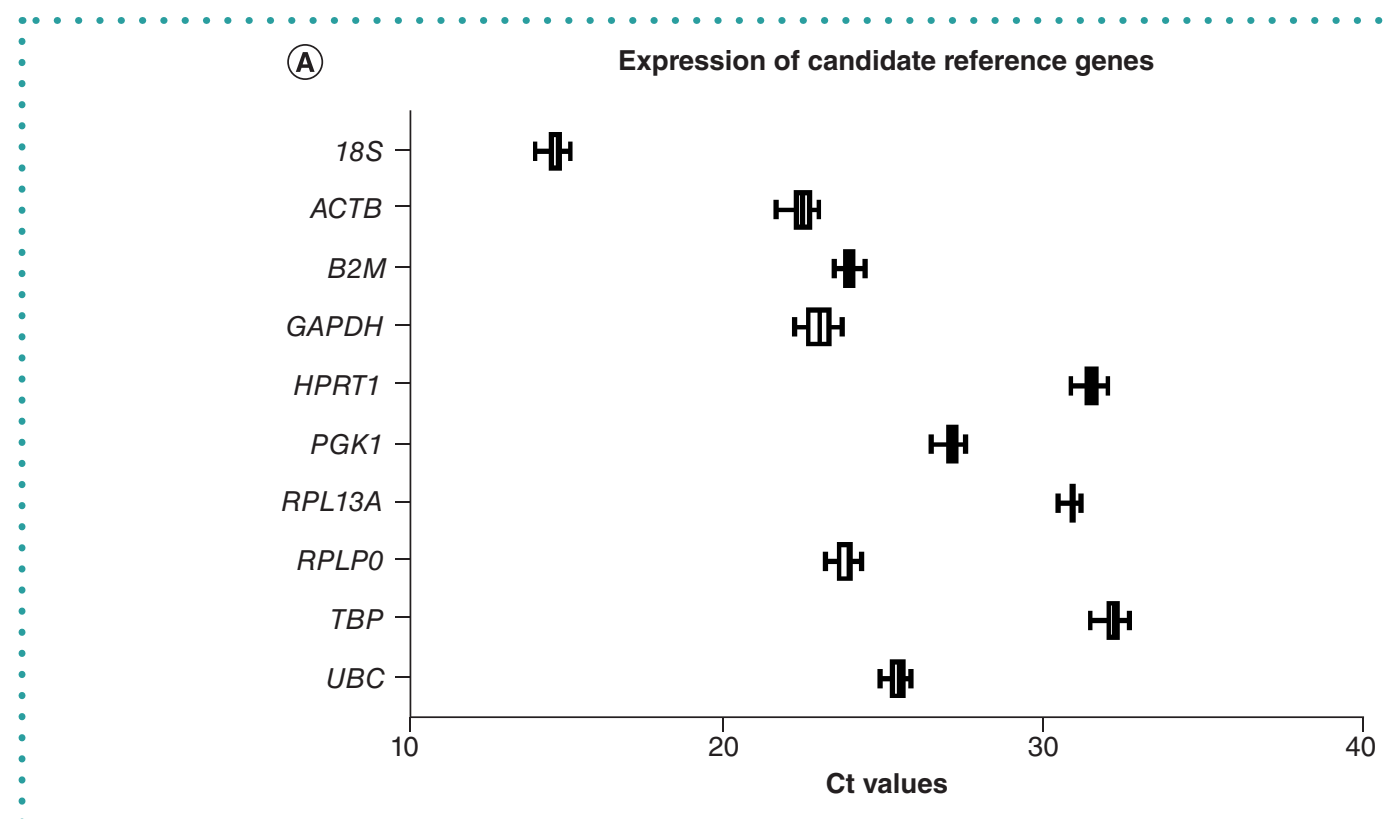

(B) Average expression stability values of remaining control genes

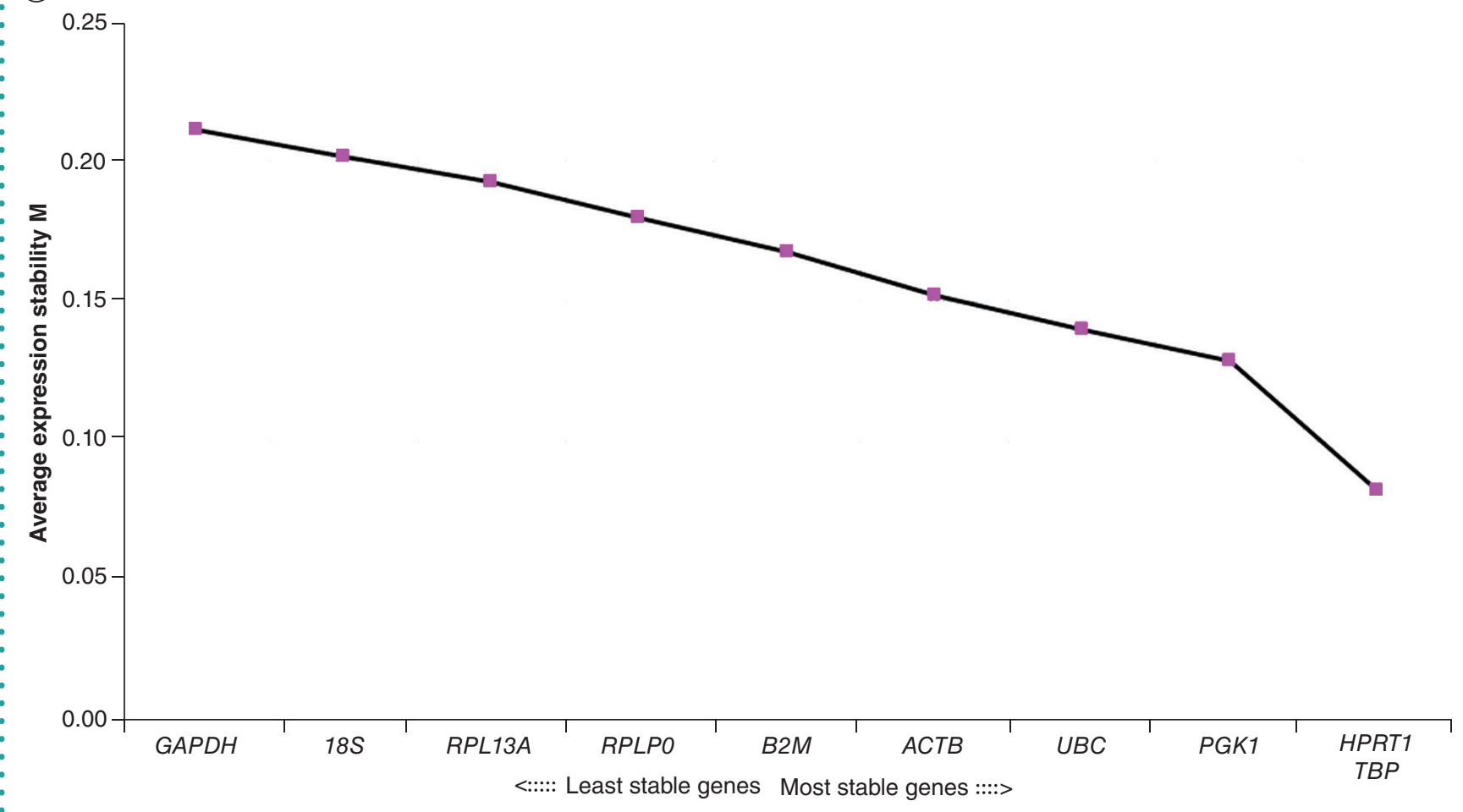

Figure 2. Analysis of reference gene stability in trypsinized versus adherent human mesenchymal stromal cells. (A) Box and whisker graphical representation of variability of $\mathrm{Ct}$ values for ten candidate reference genes. The line is the median, while the box extends from the 25th to 75 th percentiles. The whiskers range from minimum to maximum. (B) Average expression stability values of candidate reference genes calculated by geNorm.

focal adhesion, but also other key signaling pathways are affected, such as autophagy, PI3K-ATK and insulin signaling.

The differential expression of two miRNAs was confirmed by qPCR: miR-1246 (downregulated in trypsinized cells) and miR-16-1-3p (upregulated in trypsinized cells). miR-1246 is generally regarded as having a prominent role in tumor initiation, progression or maintenance of cancer stemness in non-smallcell lung cancer together with miR-1290 (also downregulated in 3D samples) [38], oral carcinoma [39] and multiple myeloma [40], among others. Moreover, it has been reported to be upregulated in aggressive breast cancer subtypes and in the associated MSCs of the tumor microenvironment [41], promoting inflammation through regulation of the NF- $\mathrm{KB}$ signaling pathway. On the contrary, miR-16-1-3p, which is upregulated in trypsinized cells, is usually reported as an oncosuppressive miR often downregulated or 


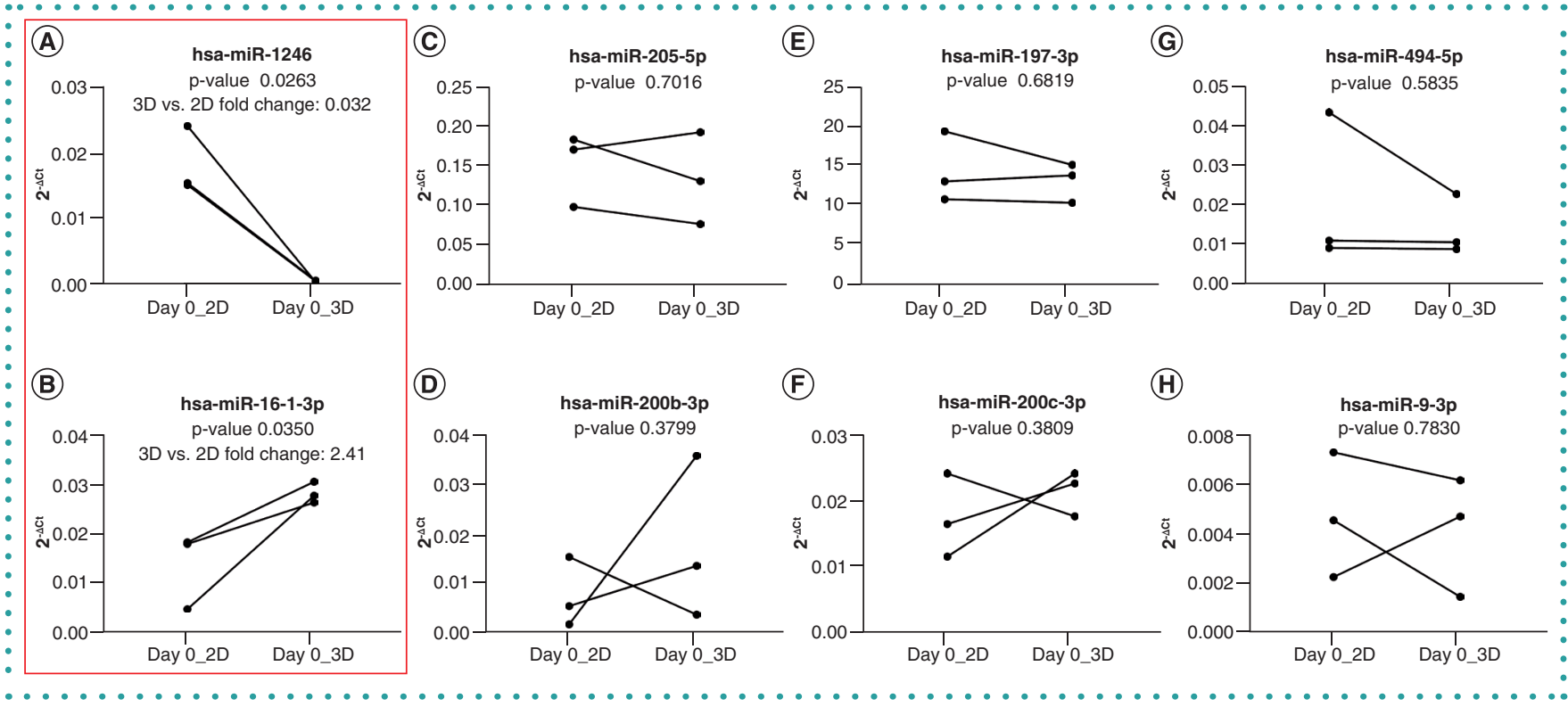

Figure 3. Validation of differential microRNA expression in trypsinized versus adherent human mesenchymal stromal cells. (A) hsa-miR-1246. (B) hsa-miR-16-1-3p. (C) hsa-miR-205-5p. (D) hsa-miR-200b-3p. (E) hsa-miR-197-3p. (F) hsa-miR-200c-3p. (G) hsa-miR-494-5p. (H) hsa-miR-9-3p. Results of trypsinized cells (day 0_3D) versus monolayer (day 0_2D) samples were compared with a two-tailed unpaired Student's t-test. The p-value for each comparison is reported, while the fold change (3D vs 2D) is indicated for hsa-miR-1246 and hsa-miR-16-1-3p. The lines indicate the trend of expression of a single donor. Each dot represents the average of the three samples derived from each single donor. The red box highlights the miRNAs that resulted significantly different in trypsinized versus monolayer cells.

$\checkmark$ deleted in several tumors such as osteosarcoma [42] or chronic lymphocytic leukemia [43]. Its upregulation results in apoptosis and proliferation inhibition via the p53 signaling pathway, as shown in chicken myoblasts [44]. Altogether, the results on the differential regulation of miR-1246 and miR-16-1-3p indicate that they may be part of a physiological mechanism of control leading MSCs to cell death after loss of anchorage, also known as anoikis [45], with important implica-

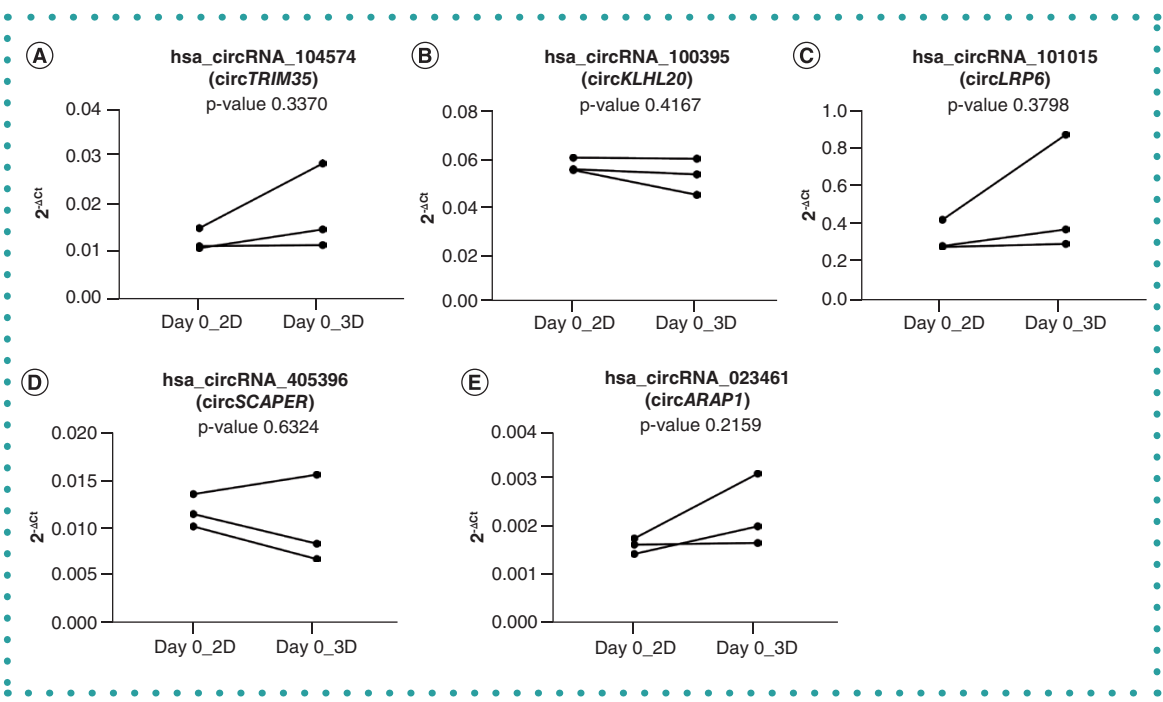

Figure 4. Validation of differential circular RNA expression in trypsinized versus adherent human mesenchymal stromal cells. (A) hsa_circRNA_104574. (B) hsa_circRNA_100395. (C) hsa_ circRNA_101015. (D) hsa_circRNA_405396. (E) hsa_circRNA_023461. Results of trypsinized cells (day 0_3D) versus monolayer (day 0_2D) samples were compared with a two-tailed unpaired Student's t-test. The $p$-value and fold change for each comparison is reported. The lines indicate the trend of expression of a single donor. Each dot represents the average of the three samples derived from each single donor.

tions for the study of cancer initiation for MSC-derived tumors such as osteosarcoma or Ewing's sarcoma $[46,47]$. There are several miRNAs with a known role in anoikis or in the Hippo pathway. It has been reported that YAP1 inactivation is required for anoikis in noncancer cells, and cell detachment in immortalized human breast epithelial cells can lead to YAP1 inactivation to induce anoikis [3]. Some of the miRNAs that were previously suggested to be associated to anoikis or Hippo pathways (miR-200b-3p, miR-200c-3p, miR-494-5p, miR-9-3p and miR-429) also appeared to be differentially regulated in $3 \mathrm{D}$ versus $2 \mathrm{D}$ by RNAseq analysis [3,48]; however, the differential expression of those targets was not confirmed by qPCR analysis.

For specific pi-and circRNAs, studying their role in certain conditions is still hampered by the paucity of powerful bioinformatics tools and databases available. Moreover, a standard and unambiguous nomenclature for both pi- and circRNA is urgently needed for a better comparison of results across different studies using different platforms or reference databases. For circRNA, a promising attempt in this 
direction is represented by circbank [49], which suggests a novel naming system and collects known features of circRNAs such as miRNA binding sites or m6A modification.

The identification of piRNA targets is of essential importance to clarify their role in physiology and disease. At least four of the upregulated piRNAs target transposable elements: piR-hsa-27489, piR-hsa30374, piR-hsa-25786 and piR-hsa-20613. Sequence alignment with the NCBI Nucleotide BLAST tool [50] using the accession number as a query against the human nucleotide collection ( $\mathrm{nr} / \mathrm{nt}$ ) for the full dataset revealed that the potential targets of piRNA are manifold, ranging from transposable elements to tRNAs, rRNAs and mRNAs, confirming their important role not only in protecting the integrity of germline cell genomes, but also in somatic cells with regulatory properties.

For circRNA, we have designed primers that could specifically detect the expression of the circular transcript by either a divergent primer strategy or with one of the primers recognizing the backspliced junction. However, the differential expression of a selection of circRNAs was not confirmed by qPCR analysis. It should be noted that the FDR for all the circular RNAs was very high ( $>0.99$ ), thus the probability of those results being true positives was low. This confirms, if necessary, the importance of validation experiments when approaching high-throughput techniques such as RNAseq or RNA hybridization. Nonetheless, we were able to confirm the expression of those circular RNA in MSCs, with at least one (hsa_circRNA_101015 derived from the LRP6 gene) being expressed at high levels in comparison to the reference gene used. A more in-depth study may be helpful to understand its role in the regulation of the Wnt pathway in MSCs, since LRP6 is a receptor for Wnt ligands for canonical $\beta$-catenindependent signaling.

Another factor that can be involved in transcriptomic changes, beside loss of anchorage, is the stress induced by the trypsinization process itself (chemical action of trypsin, cellular stress induced by shear forces and centrifugation). It has been reported that the use of trypsin can affect
RNA integrity due to the presence of ribonucleases derived from pancreas [51], thus arguing against detachment if not needed for specific research questions.

Finally, but no less important, we have identified a set of suitable reference genes that can be used for normalization of gene expression data in this experimental setting. Indeed, their expression remains unvaried regardless of adhesion status. Stability analysis has revealed that the selected 10 genes are not massively regulated within 30 min of detachment from the substrate. The three most stable genes have been also identified (HPRT1, $P G K 1$ and TBP), which could be useful for studies involving loss of anchorage or when detachment of cells cannot be avoided. A similar approach was used to determine whether the expression of two miRNAs, that is, hsa-miR-361-5p and miR-186-5p, could be used for normalization purposes, and we finally used the geometric mean of their $\mathrm{Ct}$ values for the calculation of relative gene expression. As for circular RNA expression analysis, we normalized their expression to RPLPO measured in the same samples and with the same chemistry of detection (SYBR green), since this gene also showed low stability values. We also analyzed the expression of HPRT1, $P G K 1$ and TBP in RNase-treated samples and used their geometric mean for the $\Delta \mathrm{Ct}$ calculation (data not shown); we observed that the final results did not significantly differ when using RPLPO. Thus, since the three reference genes used TaqMan chemistry while RPLPO could be used in SYBR green, we believe that in our specific case it was more appropriate to use RPLPO.

In conclusion, the data presented may represent effects induced by either trypsinization or anchorage loss (but enriched pathways are related to adhesion). However, it is evident that alterations in levels of noncoding RNAs are rapidly induced, affecting a wide variety of critical pathways. This should be carefully considered for a proper design and run of experiments.

\section{FUTURE PERSPECTIVE}

The functional validation of the aforementioned targets is the next step to further understand the changes involved in loss of adhesion of hMSCs. In addition, investi- gating wider time frames and different methods of detachment will provide a more complete picture on this topic. This is not only important for addressing technical issues and for a proper cell data analysis, but also for research in different fields.

\section{SUPPLEMENTARY DATA}

To view the supplementary data that accompany this paper please visit the journal website at: www.future-science. com/doi/suppl/10.2144/btn-2019-0038

\section{AUTHOR CONTRIBUTIONS}

EDB: Experimental design, sample preparation, data collection and analysis, manuscript writing. MJS: Conceptualization, experimental design, supervision, manuscript revision and approval.

\section{ETHICAL CONDUCT OF RESEARCH}

The authors state that they have obtained appropriate institutional review board approval or have followed the principles outlined in the Declaration of Helsinki for all human or animal experimental investigations. In addition, for investigations involving human subjects, informed consent has been obtained from the participants involved.

\section{DATA DEPOSITION}

Data are available in Gene Expression Omnibus (GEO) database with the following accession numbers: GSE129345, GSE129350 and GSE129357.

\section{FINANCIAL \& COMPETING INTERESTS DISCLOSURE}

This Investigation was financially supported with the assistance of the AO Foundation via an AOTRAUMA Network grant. The authors have no other relevant affiliations or financial involvement with any organization or entity with a financial interest in or financial conflict with the subject matter or materials discussed in the manuscript apart from those disclosed.

No writing assistance was utilized in the production of this manuscript.

\section{OPEN ACCESS}

This work is licensed under the AttributionNonCommercial-NoDerivatives 4.0 Unported License. To view a copy of this license, visit http://creativecommons.org/licenses/ by-nc-nd/4.0/ 


\section{REFERENCES}

1. Ren X-D, Wang R, Li Q, Kahek LaF, Kaibuchi K, Clark RaF. Disruption of Rho signal transduction upon cell detachment. J. Cell Sci. 117(16), 3511 (2004)

2. Danhier P, Copetti T, De Preter G et al. Influence of cell detachment on the respiration rate of tumor and en dothelial cells. PLOS ONE 8(1), e53324-e53324 (2013).

3. Zhao B, Li L, Wang L, Wang CY, Yu J, Guan KL. Cell detachment activates the Hippo pathway via cytoskeleton reorganization to induce anoikis. Genes Dev. 26(1) 54-68 (2012)

4. Ihalainen TO, Aires L, Herzog FA, Schwartlander R, Moeller J, Vogel V. Differential basal-to-apical accessibility of lamin A/C epitopes in the nuclear lamina regulated by changes in cytoskeletal tension. Nat. Mater. 14(12) 1252-1261 (2015).

5. Kim D-H, Wirtz D. Cytoskeletal tension induces the polarized architecture of the nucleus. Biomaterials 48, 161-172 (2015).

6. Tsuji K, Ojima M, Otabe K et al. Effects of different cell-detaching methods on the viability and cell surface antigen expression of synovial mesenchymal stem cells. Cell Transplant. 26(6), 1089-1102 (2017).

7. Chen S, So EC, Strome SE, Zhang X. impact of detachment methods on M2 macrophage phenotype and function. J. Immunol. Methods 426, 56-61 (2015).

8. Bartel DP. MicroRNAs: genomics, biogenesis, mechanism, and function. Cell 116, 281-297 (2004)

9. Lau NC, Lim LP, Weinstein EG, Bartel DP. An abundant class of tiny RNAs with probable regulatory roles in Caenorhabditis elegans. Science 294(5543), 858 (2001).

10. Friedman RC, Farh KK, Burge CB, Bartel DP. Most mammalian mRNAs are conserved targets of microRNAs. Genome Res. 19(1), 92-105 (2009).

11. Esteller M. Non-coding RNAs in human disease. Nat Rev. Genet. 12, 861 (2011)

12. Chang T-C, Mendell JT. microRNAs in vertebrate physiology and human disease. Annu. Rev. Genom. Hum. G. 8(1) 215-239 (2007)

13. Iwasaki YW, Siomi MC, Siomi H. PIWI-Interacting RNA its biogenesis and functions. Annu. Rev. Biochem. 84, 405-433 (2015)

14. Robine N, Lau NC, Balla S et al. A broadly conserved pathway generates $3^{\prime} U T R$-directed primary piRNAs. Curr. Biol. 19(24), 2066-2076 (2009).

15. Yuan J, Zhang P, Cui $Y$ et al. Computational identification of piRNA targets on mouse mRNAs. Bioinformatics 32(8), 1170-1177 (2016).

16. Zhang P, Kang JY, Gou LT et al. MIWI and piRNA-mediated cleavage of messenger RNAs in mouse testes. Cell Res. 25(2), 193-207 (2015).

17. Han YN, Li Y, Xia SQ, Zhang YY, Zheng JH, Li W. PIW proteins and PIWI-interacting RNA: emerging roles in cancer. Cell. Physiol. Biochem. 44(1), 1-20 (2017).

18. Juliano $\mathrm{C}$, Wang J, Lin $\mathrm{H}$. Uniting germline and stem cells: the function of Piwi proteins and the piRNA pathway in diverse organisms. Annu. Rev. Genet. 45 447-469 (2011)

19. Van Wolfswinkel JC. Piwi and potency. PIWI proteins in animal stem cells and regeneration. Integr. Comp. Biol. 54(4), 700-713 (2014).

20. Salzman J, Chen RE, Olsen MN, Wang PL, Brown PO. Cell-type specific features of circular RNA expression. PLoS Genet 9(9), e1003777 (2013).

21. Huang S, Yang B, Chen BJ et al. The emerging role of circular RNAs in transcriptome regulation. Genomics 109(5), 401-407 (2017)

22. Legnini I, Di Timoteo G, Rossi F et al. Circ-ZNF609 is a circular RNA that can be translated and functions in myogenesis. Mol. Cell 66(1), 22-37.e29 (2017)

23. Vandesompele J, De Preter K, Pattyn F et al. Accurate normalization of real-time quantitative RT-PCR data by geometric averaging of multiple internal control genes. Genome Biol. 3(7), research0034.0031-0034.0011 (2002).

24. Gardner OFW, Alini M, Stoddart MJ. Mesenchymal stem cells derived from human bone marrow. In: Cartilage Tissue Engineering: Methods and Protocols. Doran PM (Ed.). Springer, NY, USA, 41-52 (2015)

25. piRBase. http://www.pirbase.org/database/piRNA/

26. Cepollaro S, Della Bella E, De Biase D, Visani M, Fini M. Evaluation of RNA from human trabecular bone and identification of stable reference genes. J. Cell. Physiol. 233(6), 4401-4407 (2018).

27. Andersen $\mathrm{CL}$, Jensen JL, Ørntoft TF. Normalization of real-time quantitative reverse transcription-PCR data: model-based variance estimation approach to identify genes suited for normalization, applied to bladder and colon cancer data sets. Cancer Res. 64, 5245-5250 (2004).
28. Silver N, Best S, Jiang J, Thein SL. Selection of housekeeping genes for gene expression studies in human reticulocytes using real-time PCR. BMC Mol. Biol. 7, 33 (2006).

29. Ye J, Coulouris G, Zaretskaya I, Cutcutache I, Rozen S, Madden TL. Primer-BLAST: a tool to design target-specific primers for polymerase chain reaction. $B M C$ Bioinformatics 13(1), 134 (2012).

30. Identification of differentially-expressed miRNAs after human bone marrow-mesenchymal stromal cells (hMSCs) detachment. https://www.ncbi.nlm.nih.gov/geo/ query/acc.cgi?acc $=\mathrm{GSE} 129345$

31. Girard A, Sachidanandam R, Hannon GJ, Carmell MA. A germline-specific class of small RNAs binds mammalian Piwi proteins. Nature 442, 199 (2006).

32. Roovers Elke F, Rosenkranz D, Mahdipour M et al. Piwi proteins and piRNAs in mammalian oocytes and early embryos. Cell Rep. 10(12), 2069-2082 (2015).

33. Huang $\mathrm{G}, \mathrm{Hu} \mathrm{H}$, Xue $X$ et al. Altered expression of piR$N A s$ and their relation with clinicopathologic features of breast cancer. Clin. Transl. Oncol. 15(7), 563-568 (2013).

34. Chu H, Hui G, Yuan L et al. Identification of novel piRNAs in bladder cancer. Cancer Lett. 356(2, Part B), 561-567 (2015).

35. Li Y, Wang H-Y, Wan F-C et al. Deep sequencing analysis of small non-coding RNAs reveals the diversity of microRNAs and piRNAs in the human epididymis. Gen 497(2), 330-335 (2012)

36. Identification of differentially-expressed piRNAs after human bone marrow-mesenchymal stromal cells (hMSCs) detachment. https://www.ncbi.nlm.nih.gov/ geo/query/acc.cgi?acc=GSE129350

37. Identification of differentially-expressed circular RNAs after human bone marrow-mesenchymal stromal cells (hMSCs) detachment. https://www.ncbi.nlm.nih.gov/ geo/query/acc.cgi?acc=GSE129357

38. Zhang WC, Chin TM, Yang $\mathrm{H}$ et al. Tumour-initiating cell-specific miR-1246 and miR-1290 expression converge to promote non-small cell lung cancer progression. Nat. Commun. 7, 11702 (2016)

39. Lin S-S, Peng C-Y, Liao Y-W, Chou M-Y, Hsieh P-L, Yu C-C. miR-1246 targets CCNG2 to enhance cancer stem ness and chemoresistance in oral carcinomas. Cancers (Basel) 10(8), (2018).

40. Ren Y, Li X, Wang W, He W, Wang J, Wang Y. Expression of peripheral blood miRNA-720 and miRNA-1246 can be used as a predictor for outcome in multiple myelom patients. Clin. Lymphoma Myeloma Leuk. 17(7), 415-423 (2017).

41. Bott A, Erdem N, Lerrer $S$ et al. miRNA-1246 induces pro-inflammatory responses in mesenchymal stem/ stromal cells by regulating PKA and PP2A. Oncotarget 8(27), 43897-43914 (2017).

42. Maximov VV, Akkawi R, Khawaled S et al. MiR-16-1-3p and miR-16-2-3p possess strong tumor suppressive and antimetastatic properties in osteosarcoma. Int. J. Cancer doi:10.1002/ijc.32368 (2019) (Epub ahead of print).

43. Calin GA, Cimmino A, Fabbri M et al. MiR-15a and miR16-1 cluster functions in human leukemia. Proc. Nat Acad. Sci. USA 105(13), 5166 (2008)

44. Cai B, Ma M, Chen B et al. MiR-16-5p targets SESN1 to regulate the $\mathrm{p} 53$ signaling pathway, affecting myoblast proliferation and apoptosis, and is involved in myoblast differentiation. Cell Death Dis. 9(3), 367 (2018).

45. Frisch SM, Screaton RA. Anoikis mechanisms. Curr Opin. Cell Biol. 13, 555-562 (2001).

46. Riggi N, Cironi L, Provero P et al. Development of Ewing's sarcoma from primary bone marrow-derived mesenchymal progenitor cells. Cancer Res. 65(24), 11459-11468 (2005).

47. Zheng Y, Wang G, Chen R, Hua Y, Cai Z. Mesenchymal stem cells in the osteosarcoma microenvironment: their biological properties, influence on tumor growth, and therapeutic implications. Stem Cell. Res. Ther. 9(1) 22 (2018).

48. Malagobadan S, Nagoor NH. Evaluation of MicroRNAs regulating anoikis pathways and its therapeutic potenregulating anoikis pathways and its ther
tial. BioMed Res. Int. 2015, 1-10 (2015).

49. Liu M, Wang Q, Shen J, Yang BB, Ding X. Circbank: a comprehensive database for circRNA with standard nomenclature. RNA Biol. 16(7), 899-905 (2019).

50. Standard Nucleotide BLAST. https://blast.ncbi.nlm.nih gov/Blast.cgi?PAGE=Nucleotides\&PROGRAM=blastn\&PAGE_TYPE=BlastSearch\&BLAST_SPEC=

51. VrtaČnik P, Kos Š, Bustin SA, Marc J, Ostanek B, Influence of trypsinization and alternative procedures for cell preparation before RNA extraction on RNA integrity. Anal. Biochem. 463, 38-44 (2014). 